Research Paper

\title{
Clinical Significance of Female-hormones and Cytokines in Breast Cancer Patients Complicated with Aromatase Inhibitor-related Osteoarthropathy - Efficacy of Vitamin E
}

\author{
Anna Kiyomi ${ }^{\bowtie}$, Masujiro Makita², Takuji Iwase², Sachiko Tanaka1 ${ }^{1}$, Kenji Onda1 ${ }^{1}$, Kentaro Sugiyama1, \\ Hironori Takeuchi ${ }^{3}$, Toshihiko Hirano ${ }^{1}$ \\ 1. Tokyo University of Pharmacy and Life Sciences, Department of Clinical Pharmacology, 1432-1 Horinouchi, Hachioji, Tokyo 192-0355, Japan \\ 2. The Cancer Institute Hospital of JFCR, Department of Breast Oncology, 3-8-31 Ariake, Koto-ku, Tokyo 135-8550, Japan \\ 3. Tokyo University of Pharmacy and Life Sciences, Department of Practical Pharmacy, 1432-1 Horinouchi, Hachioji, Tokyo 192-0355, Japan
}

$\triangle$ Corresponding author: Anna Kiyomi, Department of Clinical Pharmacology, School of Pharmacy, Tokyo University of Pharmacy and Life Sciences, 1432-1 Horinouchi, Hachioji, Tokyo 192-0355, Japan. Phone: +81-42-676-5795, Fax: +81-42-676-5798, E-mail: y064076@toyaku.ac.jp

(c) 2015 Ivyspring International Publisher. Reproduction is permitted for personal, noncommercial use, provided that the article is in whole, unmodified, and properly cited. See http://ivyspring.com/terms for terms and conditions.

Received: 2014.10.01; Accepted: 2014.12.05; Published: 2015.02.25

\begin{abstract}
Introduction: Aromatase inhibitor use for postmenopausal hormone-sensitive breast cancer patients often results in drug-induced osteoarthropathy, while its accurate mechanism has not been clarified. We investigated the implication of female hormones and several cytokines in osteoarthropathy complicated with aromatase inhibitor treatment, and the efficacy of vitamin $E$ on the severity of osteoarthropathy, in breast cancer patients.

Methods: Sixty two breast cancer patients treated with aromatase inhibitor for average of 1.77 years were included. These patients were orally administered vitamin $E(150 \mathrm{mg} /$ day $)$ for 29.8 days to alleviate aromatase inhibitor-related osteoarthropathy. Severity of osteoarthropathy was scored, and the patients were grouped based on the severity or vitamin E efficacy. Serum estradiol, progesterone, vitamin $\mathrm{E}$, interferon-gamma (IFN-gamma), tumor necrosis factor-alpha (TNF-alpha), IL-2, IL-4, IL-6, IL-10, and IL-17A concentrations were measured by ELISA or beads array procedures followed by flow cytometry.

Results: There was no significant difference in serum concentrations of the biomarkers between the severe and the mild osteoarthropathy groups before vitamin E administration. The osteoarthropathy scores significantly decreased after vitamin $E$ administration $(p=0.0243)$, while serum-estradiol concentrations did not change. The serum-estradiol concentrations before vitamin $E$ administration in the group sensitive to the vitamin $E$ efficacy were significantly lower, as compared with those in the insensitive group $(p=0.0005)$. The rate of the highly sensitive patients to the vitamin E efficacy in those exhibiting low serum-estradiol concentrations was significantly higher than that in the high serum-estradiol group $(p=0.0004)$. In the sensitive group, serum-estradiol concentrations after taking vitamin $\mathrm{E}$ were significantly higher than those before taking vitamin $E(p=0.0124)$.

Conclusions: Vitamin E administration seemed to be a potential way for relieving osteoarthropathy complicated with aromatase inhibitor use. Using serum-estradiol concentration, it would be possible to select out the breast cancer patients who will respond well to the vitamin $E$ therapy for osteoarthropathy complicated with aromatase inhibitor.
\end{abstract}

Key words: aromatase inhibitor-related osteoarthropathy, breast cancer, vitamin E, serum female hormones, serum cytokines 


\section{Introduction}

Aromatase inhibitors, such as anastrozole, exemestane, and letrozole, which inhibit estradiol biosynthesis, are used for treatment of postmenopausal hormone-sensitive breast cancer patients. However, aromatase inhibitor use often results in drug-induced osteoarthropathy as a major side effect. Likewise, clinical experience also suggests that aromatase inhibitor therapy is associated with a novel musculoskeletal side effect consisting of an arthralgia syndrome [1], which would be a reason for discontinuation of aromatase inhibitor treatment. Because osteoarthropathy frequently limits activity of daily living, quality of life of breast cancer patients will decline necessarily. However, the accurate mechanism underlying the occurrence of osteoarthropathy accompanied by aromatase inhibitor treatment has not been clarified. Despite the proposal such as physical therapy or analgesic drug use, there is no optimal treatment which supports the long-term hormone therapy [2].

We have suggested in our pilot study that vitamin $\mathrm{E}$ is effective in improving osteoarthropathy complicated with aromatase inhibitor treatment for breast cancer patients, in which we observed palliative efficacy of vitamin $\mathrm{E}$ on aromatase inhibitor induced osteoarthropathy in 22 out of 28 breast cancer patients [3]. Vitamin $\mathrm{E}$ has been known to have membrane-stabilizing action and anti-oxidative efficacy [4], and is used for treatment of peripheral circulatory disturbance such as menopausal symptom, feeling of cold, and numbness [5]. According to these observations, vitamin $\mathrm{E}$ is suggested to be useful for treatment of osteoarthropathy. Vitamin $\mathrm{E}$ is one of the essential factors, and therefore it might have few adverse effects in human. Whereas, there are concerns that vitamin E may influence the environment of female hormones.

It is reported that serum IL-6 level decreases accompanying with the decrease of estradiol in postmenopausal women [6]. Estrogen deficiency was also found to induce differentiation of Th17 cells secreting an inflammatory cytokine IL-17 [7]. Thus, modulation of immune system resulted from changes in hormone balance may have influence on the onset and/or pathogenesis of aromatase inhibitor induced osteoarthropathy.

Then, in this study, we investigated the implication of female hormones and several cytokines in osteoarthropathy complicated with aromatase inhibitor treatment in breast cancer patients. Furthermore, the effects of vitamin $\mathrm{E}$ on the severity of osteoarthropathy, serum hormone levels, and serum concentrations of several Th1/Th2/Th17 cytokines were examined in these patients.

\section{Materials and methods}

\section{Patients and osteoarthropathy scoring}

This study was approved be Ethical Committee of the Cancer Institute Hospital of JFCR (UMIN000004416). Informed consent was obtained from all the patients before enrolling participants in this study. We evaluated totally 62 postmenopausal breast cancer patients over 50 years old who attended the Cancer Institute Hospital and were taking one of the following aromatase inhibitors; anastrozole, exemestane, or letrozole, as the supportive endocrine therapy (Table 1). All of these patients had been surgically resected their breast cancer, and were received adjuvant chemotherapy and/or radiation therapy. They complained of arthralgia over Grade 2 (moderate pain, which means pain or analgesics interfering with function, but not interfering with activities of daily living) (NCI-CTC Version2.0) after the starting of aromatase inhibitor treatment. Exclusion criteria were applied for the patients who had pre-existing arthropathy such as chronic rheumatism, osteoarthritis, shoulder periarthritis, severe osteoporosis, past history of mental disorder, and for those previously taking vitamin $\mathrm{E}$ as supplement. Patients taking anticoagulant or the patients who have experience receiving surgery other than breast cancer resection were also excluded. The patients were treated with aromatase inhibitor for an average of $1.77 \pm 0.16$ years. These patients were orally administered $150 \mathrm{mg} /$ day tocopherol acetate (Eisai Inc., Japan) for an average of $29.8 \pm 0.34$ days to alleviate aromatase inhibitor-related osteoarthropathy. Before and after administration of vitamin E, peripheral blood sampling and questionnaire were carried out. Osteoarthropathy symptoms were scored as 0 (no symptom) to 3 (worse possible symptom) for each of stiffness and low back pain. Then, the total score was calculated for each patient based on these scores, and they were grouped into the mild group and the severe group by threshold of median score (the median score was 4). Similarly, the patients were divided into two groups namely the sensitive and the insensitive groups based on the criteria for their responses to vitamin $\mathrm{E}$ with the improvement scores of the better (the scores were reduced) or the stable/worse (the scores were unchanged or increased).

\section{Hormones and vitamin E measurements}

Nine $\mathrm{ml}$ peripheral blood was collected from the patients, and the serum was isolated. Serum estradiol and progesterone concentrations were measured by electrochemiluminescence immunoassay (ECLIA) with Eclusys ${ }^{\circledR}$ agent E2 III and Progesterone II (Roche Diagnostics Co., Japan), respectively, according to the 
manufacture's instruction. Serum vitamin E concentrations were determined by measuring fluorescence intensity of the compound. In brief, $0.8 \mathrm{~mL}$ of purified water and ethanol (special grade reagent) (Wako Inc., Japan) were added to $0.5 \mathrm{ml}$ of serum, and mixed. Then, the mixture was extracted with $5 \mathrm{~mL}$ of n-hexane (special grade reagent) (Wako Inc., Japan), and the mixture was centrifuged. The n-hexane phase was collected, and then measured fluorescence intensity by spectrofluorometer (Format 650-10S, Hitachi Inc., Japan). The tocopherol emits fluorescence of $320 \mathrm{~nm}$ by excitation of $295 \mathrm{~nm}$ was used for the measurement.

Table 1. Patient Characteristics

\begin{tabular}{lll}
\hline Patient grouping based on; & Remarks & $\begin{array}{l}\text { Number of } \\
\text { Patients }\end{array}$ \\
\hline Aromatase inhibitor used & anastrozole & 50 \\
& exemestane & 2 \\
letrozole & 10 \\
BMI & Nonobesity & 50 \\
& Obesity & 12 \\
The year after post menopause & $<10$ years & 21 \\
& $\geq 10$ years & 38 \\
The year after taking aromatase inhibitor & Unknown & 3 \\
& $<2$ years & 41 \\
The osteoarthropathy symptom before vitamin & $\geq 2$ years & 21 \\
E administration & Mild & 22 \\
& Severe & 34 \\
The effect of vitamin $E^{* *}$ & Unknown & 6 \\
& Sensitive & 24 \\
& Insensitive & 27 \\
& unknown & 11 \\
\hline
\end{tabular}

*Symptoms were scored as 0-3 points ( 3 is the worst) based on NCI-CTC Version2.0 for each symptom; stiffness and low back pain. Then, the total score was calculated for each patient, and the patients were divided into mild and severe groups by the median score (median $=4)$.

**These patients were classified into two groups according to their responses to the therapeutic effect of vitamin $\mathrm{E}$.

"Unknown" patients were not received questionnaire or refused to answer some questions.

\section{Isolation of PBMCs and evaluation of drug ef- fects in vitro}

Twenty milliliters of venous blood was taken from healthy subjects $(n=4$, two male and two female, average of age was $32 \pm 13.9$ years). The heparinized blood was loaded on $3 \mathrm{~mL}$ of Ficoll-Hypaque (Nakarai Co., Japan), centrifuged at $1300 \times \mathrm{g}$ for 20 min, and PBMCs were separated. For the evaluation of the effects of anastrozole and vitamin E on PBMCs, the cells were washed and suspended in RPMI 1640 medium containing $10 \%$ fetal bovine serum, 100,000 $\mathrm{IU} / \mathrm{L}$ penicillin and $100 \mathrm{mg} / \mathrm{L}$ streptomycin to a final density of $1 \times 10^{6}$ cells $/ \mathrm{mL}$. $196 \mu \mathrm{L}$ of this cell suspension was loaded into each well of a 96-well plate. Concanavalin A, as T cell mitogen, was added to each well to a final concentration of $5.0 \mu \mathrm{g} / \mathrm{mL}$. Subsequently, $4 \mu \mathrm{L}$ of an ethanol solution containing anas- trozole (Sigma-Aldrich Co.LLC, Japan) or $(+)$ - $\alpha$-tocopherol (Iwaki\&Co., Ltd., Japan) was added to give final agent concentrations of $0.01-100 \mu \mathrm{M}$. Four microliters of ethanol was added to the control wells. The plate was incubated for $72 \mathrm{~h}$ in $5 \% \mathrm{CO}_{2} /$ air at $37^{\circ} \mathrm{C}$.

\section{Th1/Th2/Th 17 cytokine measurements}

Serum concentrations of interferon- $\gamma$ (IFN- $\gamma$ ), tumor necrosis factor- $\alpha$ (TNF- $\alpha$ ), IL-2, IL-4, IL-6, IL-10, and IL-17A were measured using BD Cytometric Bead Array (CBA) Human Th1/Th2/Th17 Cytokine Kit (BD Inc., USA). The assay procedures were carried out according to the manufacture's instruction, and the data were analyzed with BD CBA software. This assay employs a mixture of beads with distinct fluorescent intensity characteristics to simultaneously detect multiple cytokines in serum samples. Each bead is coated with an antibody directed against a distinct cytokine, and the different fluorescent signals are detected via flow cytometry. The kit performance has been optimized for analysis of physiologically relevant concentrations of cytokines over a broad dynamic range [8].

\section{Statistical Analysis}

Statistical significance for the difference of concentrations of the biomarkers; vitamin E, estradiol, progesterone, and cytokines in serum between any two patient subgroups was analyzed using the Mann Whitney's U-test. The paired $t$ test was used to compare the osteoarthropathy scores and serum concentrations of biomarkers between patients before and after administration of vitamin E. The correlation between serum concentrations of estradiol and each cytokine or between osteoarthropathy improvement rates after vitamin $\mathrm{E}$ therapy and the biomarker concentrations in serum before administration of vitamin E was analyzed by the Pearson's correlation coefficient. The correlation between concentrations of serum biomarkers and the osteoarthropathy improvement scores was analyzed using the Kruskal-Wallis test. The Chi-square test was performed for analyzing difference in the frequencies of the severe osteoarthropathy subjects before vitamin $\mathrm{E}$ therapy between patients with high- and low-serum estradiol concentrations. The diagnostic performance to differentiate patients who will respond well to the vitamin $\mathrm{E}$ therapy by the serum estradiol level of $1.8 \mathrm{pg} / \mathrm{ml}$ was also confirmed by the receiver-operating characteristic (ROC) analysis between serum estradiol concentrations and the vitamin $E$ effectiveness. $P$ values less than 0.05 were estimated to be statistically significant. All statistical analysis, except for the ROC analysis, was done by GraphPad PRISM 4.0 (GraphPrism 
software Inc., USA). JMP version 8.0.2 (SAS Institute Inc., USA) was used for the ROC analysis.

\section{Results}

\section{Serum concentrations of estradiol, proges- terone, vitamin E, and Th1/Th2/Th 17 cyto- kines in breast cancer patients treated with aromatase inhibitor}

We compared the differences in serum concentrations of several biomarkers between two patient groups; the mild osteoarthropathy group $(n=22)$ and the severe osteoarthropathy group $(n=34)$, complicated with aromatase inhibitor treatment. Evaluation of osteoarthropathy scores in 6 patients has not been completed, and these patients were not included in this examination. There was no significant difference in the concentrations of estradiol, progesterone, vita$\min \mathrm{E}, \mathrm{IFN}-\gamma, \mathrm{TNF}-\alpha$, IL-2, IL-4, IL-6, IL-10, and IL-17A between the two patient groups (data not shown). We further evaluated relationships between serum cytokine concentrations and the hormone levels. There was a significant positive correlation between serum estradiol concentrations and IL-17A concentrations $(p=0.0291)$ (Figure 1). Whereas, there were no significant correlations between serum concentrations of progesterone, vitamin E or Th1/2 cytokines, and those of estradiol (data not shown).

\section{Influence of vitamin E administration on the osteoarthropathy complication scores and serum biomarkers}

The therapeutic efficacies of vitamin $\mathrm{E}$ to reduce scores of osteoarthropathy were investigated in 51 patients who could be evaluated the osteoarthropathy scores at both before and after the vitamin $\mathrm{E}$ admin-

\section{A) Score}

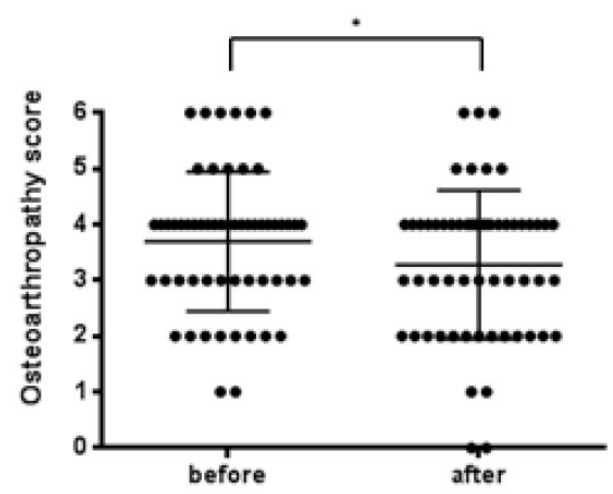

istration. The mean osteoarthropathy scores related to aromatase inhibitor treatment significantly decreased after administration of vitamin $\mathrm{E}(p=0.0178)$ (Figure 2A).

In order to confirm whether vitamin $E$ influence serum estradiol level of breast cancer patients taking aromatase inhibitor, we measured the hormone concentrations before and after the vitamin E administration. There was no significant change in the mean estradiol concentrations after administration of vitamin $\mathrm{E}$ (Figure 2B). Whereas, the serum concentrations of vitamin $\mathrm{E}(p<0.0001)$ significantly increased after the administration of vitamin E (Figure 3). Moreover, we investigated the correlations between osteoarthropathy scores and the serum concentrations of several biomarkers described above. There was no significant correlation between serum concentrations of several biomarkers examined and the scores, neither before nor after administration of vitamin $\mathrm{E}$ (data not shown).

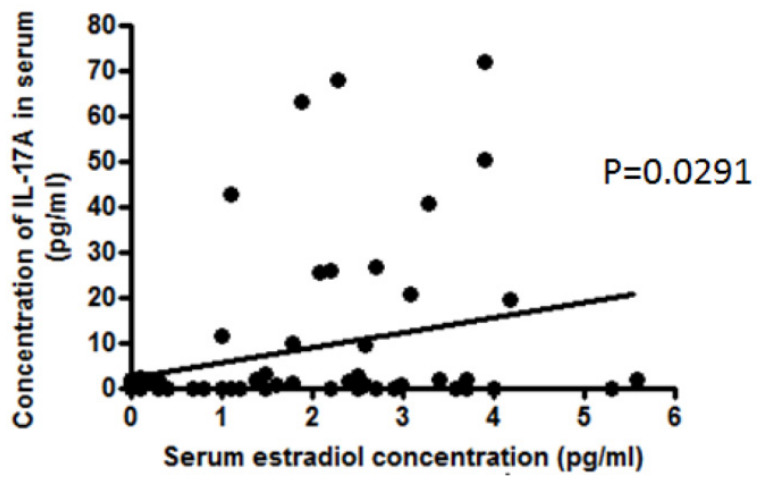

Figure 1. The correlation between serum estradiol concentrations and IL-17A concentrations. There was a positive correlation between serum estradiol concentrations and IL-17A concentrations in 62 breast cancer patients before administration of vitamin E, as analyzed by Pearson's correlation coefficient test $(p=0.0291)$.

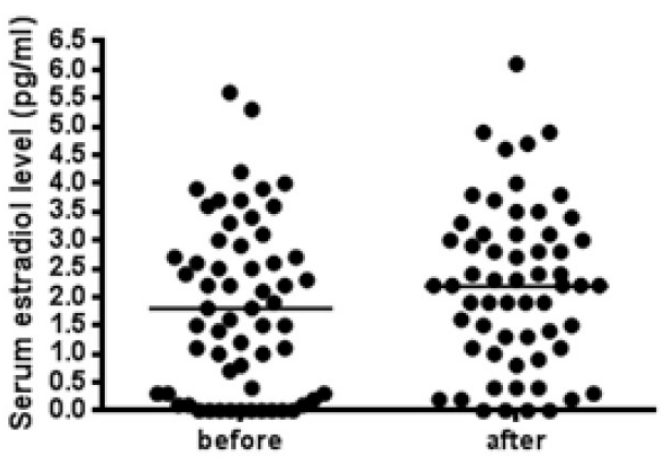

Figure 2. Osteoarthropathy scores and estradiol concentrations in aromatase inhibitor treated patients after vitamin $E$ administration $(n=60)$. (A) A statistically significant decrease in the mean scores was observed after the vitamin $\mathrm{E}$ administration, as compared to the mean scores before administration, as analyzed by Wilcoxon test $(p=0.0178)$. (B) There was no significant change in the mean estradiol concentrations after administration of vitamin $E$ by paired $t$ test. 


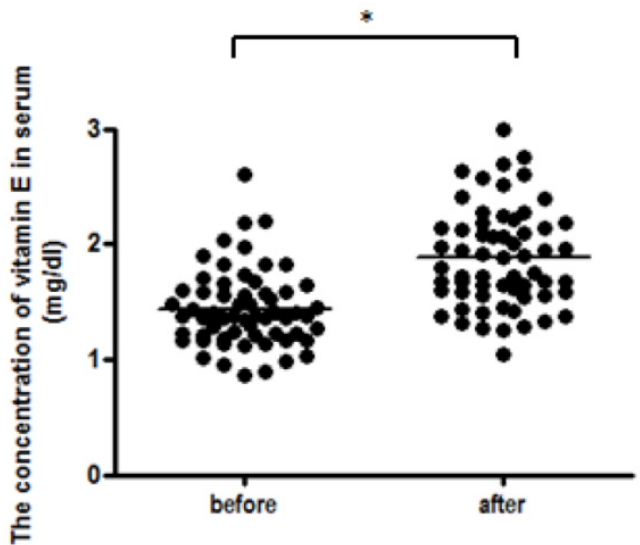

Figure 3. Changes of serum vitamin E concentrations in patients after vitamin $\mathbf{E}$ administration. Statistically significant increases in the mean concentrations of vitamin E was observed after the vitamin E administration, as compared to those before administration, as analyzed by paired $t$ test $(p<0.0001)(n=61)$.

\section{Relationship between serum estradiol con- centration and efficacy of vitamin $E$}

In order to confirm the therapeutic efficacy of vitamin $\mathrm{E}$ for osteoarthropathy complicated with aromatase inhibitor, we calculated the improvement rates of osteoarthropathy in the breast cancer patients who answered all questionnaires required for the osteoarthropathy scoring $(\mathrm{n}=51)$. The improvement rates were calculated by subtracting the scores after administration of vitamin $\mathrm{E}$ from the scores before administration, and expressed as the percentage of improvement. Then, we analyzed relationships between the osteoarthropathy improvement rates and the serum concentrations of hormones or cytokines before the vitamin E administration. There was significantly negative correlation between the rates and the serum estradiol concentrations $(p=0.0069)$ or IL-17A concentrations $(p=0.0234)$ (Figure 4).

Thus, there would be a relationship that the lower the serum concentration of estradiol in the A) Estradiol

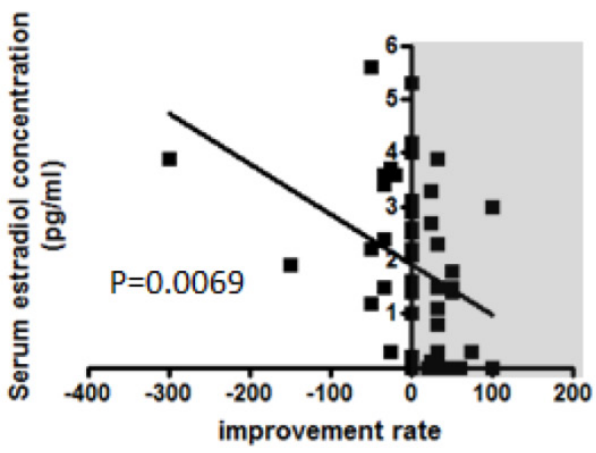

breast cancer patients taken aromatase inhibitors was, the more the therapeutic efficacy of administrating vitamin E. Accordingly, we compared serum estradiol concentrations before taking vitamin $\mathrm{E}$ between the patients who were responsive (sensitive; $n=24$ ) and the patients who were unresponsive (insensitive; $\mathrm{n}=27$ ) to the vitamin $\mathrm{E}$ therapy. The median serum estradiol concentration was significantly lower in the sensitive patients $(p=0.0005)$ (Figure 5$)$. Then, the patients were divided into the sensitive or insensitive group by the median of the osteoarthropathy scores, and the frequency of the sensitive patients was compared between any two patient subgroups with low (estradiol: $1.8 \mathrm{pg} / \mathrm{mL}$ or less, IL-17A: $0.72 \mathrm{pg} / \mathrm{ml}$ or less) and high (estradiol: more than $1.8 \mathrm{pg} / \mathrm{mL}$, IL-17A: more than $0.72 \mathrm{pg} / \mathrm{ml}$ ) concentrations of serum estradiol or IL-17A before the vitamin E administration. Serum concentrations of IL-17A before vitamin $\mathrm{E}$ administration had no significant relation with the sensitivity to vitamin E therapy (data not shown), whereas the patients whose serum estradiol concentrations were $1.8 \mathrm{pg} / \mathrm{ml}$ or less showed significantly high frequency for the subjects improving osteoarthropathy after vitamin E administration $(p=0.0004)$ (Table 2). Furthermore, a strong correlation between serum estradiol concentrations and the sensitivity to the therapeutic efficacy of vitamin E was identified, with an area under the receiver operating characteristic (ROC) curve (AUC) between the serum estradiol concentration and the vitamin $\mathrm{E}$ sensitivities of 0.78074 (Figure 6), which resulted in an optimal cutoff-point of $1.8 \mathrm{pg} / \mathrm{ml}$. When applying this discriminatory estradiol value of $1.8 \mathrm{pg} / \mathrm{ml}$, the criteria sensitivity and the specificity of the serum estradiol concentration for predicting the vitamin E efficacy was $80.0 \%$ and $70.37 \%$, respectively, with a correctly patient classification rate of $74.50 \%$.

\section{B) IL-17A}

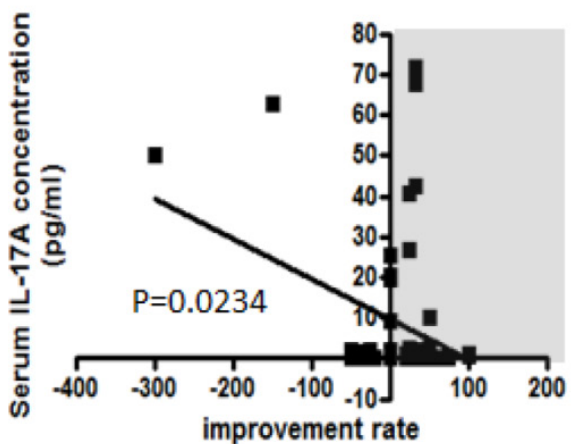

Figure 4. Correlations between osteoarthropathy improvement rates and serum estradiol or IL-17A concentrations. There were correlations between improvement rates of osteoarthropathy (\%) and serum concentrations of A) estradiol or B) IL-17A (pg/ml) in 51 breast cancer patients before administration of vitamin E. There were significant negative correlations in A) estradiol $(p=0.0069)$ and B) IL-17A $(p=0.0234)$. The plots shown in un-shaded area (left zone) represents the patients with worse symptoms after the vitamin $E$ administration, whereas the plots shown in shaded area (right zone) represents the patients with improvement in the symptoms. Zero percent in the abscissa means no change in the scores. 


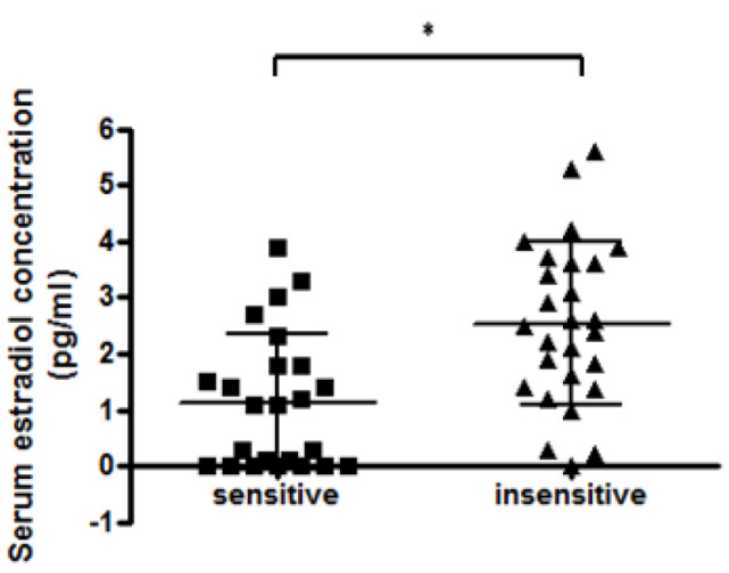

Figure 5. Comparison of serum estradiol concentrations between vitamin E sensitive or insensitive patients. Serum estradiol concentrations, before administration of vitamin $E$, were compared between the vitamin $E$ responsive patients (sensitive; $n=24$ ) and the vitamin $E$ unresponsive patients (insensitive; $n=27$ ). There was a significant difference in the median concentrations of estradiol, as analyzed by the Mann Whitney's U-test $(p=0.0005)$.

Table 2. Relationship between serum estradiol concentrations and responses to vitamin $E$ therapy.

\begin{tabular}{llll}
\hline $\begin{array}{l}\text { Response to the therapeutic } \\
\text { efficacy of vitamin E }\end{array}$ & \multicolumn{2}{l}{$\begin{array}{l}\text { Serum estradiol before vitamin } \\
\text { E administration }(\mathrm{pg} / \mathrm{mL})\end{array}$} \\
\cline { 2 - 4 } & Low: $1.8 \geq$ & High: $1.8<$ & Total \\
\hline Sensitive & $19^{*}$ & 5 & 24 \\
Insensitive & 8 & 19 & 27 \\
Total & 27 & 24 & 51
\end{tabular}

The patients were grouped into the sensitive and the insensitive patients by the median score for improvement of osteoarthropathy symptoms after the treatment by vitamin E. On the other hand, the patients were divided into the low- and high-serum estradiol concentration groups based on the median estradiol concentration of $1.8 \mathrm{pg} / \mathrm{ml}$. Consequently, the incidence of the sensitive patients to the vitamin $\mathrm{E}$ therapy was compared between the low- and high-serum estradiol concentration groups. Statistical analysis was performed by Chi-Square test* ${ }^{*}$. The patients with low concentrations of serum estradiol exhibited significantly high incidence of the sensitive subjects responding to the vitamin $\mathrm{E}$ for osteoarthropathy caused by aromatase inhibitor $(p=0.0004)$.

Fifty-one patients who answered all questionnaires were divided into the vitamin $\mathrm{E}$ sensitive and insensitive group (see Table 1), and serum estradiol concentrations between the patients before administration of vitamin $\mathrm{E}$ and the same patients after the administration were compared. After the administration of vitamin $\mathrm{E}$, the serum estradiol concentrations in all of the patients were significantly increased $(\mathrm{n}=51$, from $1.88 \pm 0.21 \mathrm{pg} / \mathrm{ml}$ to $2.38 \pm 0.20 \mathrm{pg} / \mathrm{ml}$, $p=0.0412)$. Serum estradiol concentrations in the vitamin $\mathrm{E}$ sensitive group were also increased significantly $(\mathrm{n}=24$, from $1.14 \pm 0.25 \mathrm{pg} / \mathrm{ml}$ to $2.21 \pm$ $0.32 \mathrm{pg} / \mathrm{ml}, p=0.0124)$, but the concentrations in the insensitive patients showed no significant change $(\mathrm{n}=27$, from $2.54 \pm 0.28 \mathrm{pg} / \mathrm{ml}$ to $2.53 \pm 0.24 \mathrm{pg} / \mathrm{ml})$ (Figure 7).

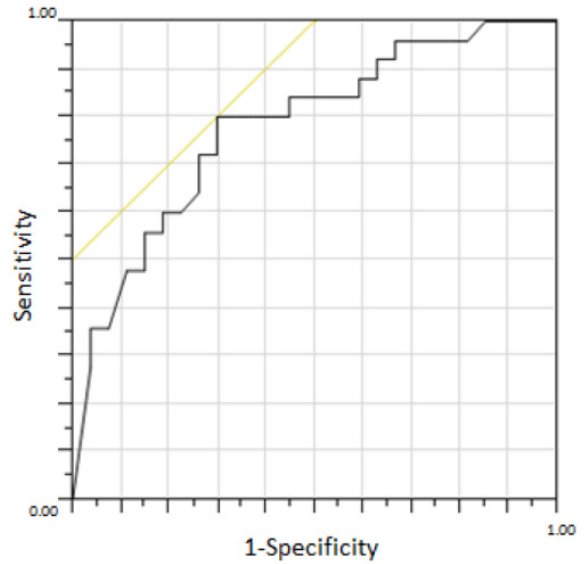

Figure 6. Serum estradiol level as a new biomarker for patients who will respond to vitamin $E$. Receiver operator characteristic (ROC) curve for serum estradiol concentrations as a function of the improvement scores for osteoarthritis by vitamin $\mathrm{E}$ treatment. The areas under the curve (AUC) was 0.78074 , and the cut-off value of estradiol was calculated as $1.80 \mathrm{pg} / \mathrm{ml}$ (see Results).

\section{Influence of vitamin $E$ and aromatase inhibitor on cytokine release from mitogen activat- ed-PBMCs in vitro}

In order to investigate the influence of vitamin $\mathrm{E}$ and aromatase inhibitor on PBMCs, PBMCs obtained from health subjects were cultured in the presence of serial concentrations of anastrozole $(1-100 \mathrm{ng} / \mathrm{mL})$ and/or vitamin E (1.88-188 $\mu \mathrm{g} / \mathrm{mL})$, under the stimulation with a $\mathrm{T}$ cell mitogen concanavalin A. Then, PBMC proliferation as assessed by the WST- 8 assay value and the concentrations of IFN- $\gamma$, TNF- $\alpha$, IL-2, IL-4, IL-6, IL-10, and IL-17A in the culture supernatant were measured (Figure 8). As shown in Figure 8, the mean WST-8 assay value of PBMCs treated with 10 $\mathrm{ng} / \mathrm{ml}$ anastrozole and $18.8 \mu \mathrm{g} / \mathrm{ml}$ vitamin $\mathrm{E}$ in combination was significantly higher than that of PBMCs treated with $10 \mathrm{ng} / \mathrm{ml}$ anastrozole alone $(p<0.05)$. On the other hand, anastrozole, vitamin $\mathrm{E}$, and their combination had no significant effect on the release of 7 kinds of cytokines from the activated PBMCs.

\section{Discussion}

Aromatase inhibitor therapy for postmenopausal breast cancer patients involves osteoarthropathy frequently. This complication sometimes results in dropout of the patients from aromatase inhibitor treatment. On the other hand, postmenopausal osteoporosis is possibly caused by deficiency of estradiol. Likewise, aromatase inhibitor causes reduction of estradiol level, which may result in induction of osteoarthropathy. Binding of estradiol to its receptor in osteoclast is known to activate the gene expression of Fas ligand, a factor induces apoptosis and controls lifetimes of osteoclast [9]. Therefore, the deficiency of estradiol leads to imbalance in bone metabolism. 

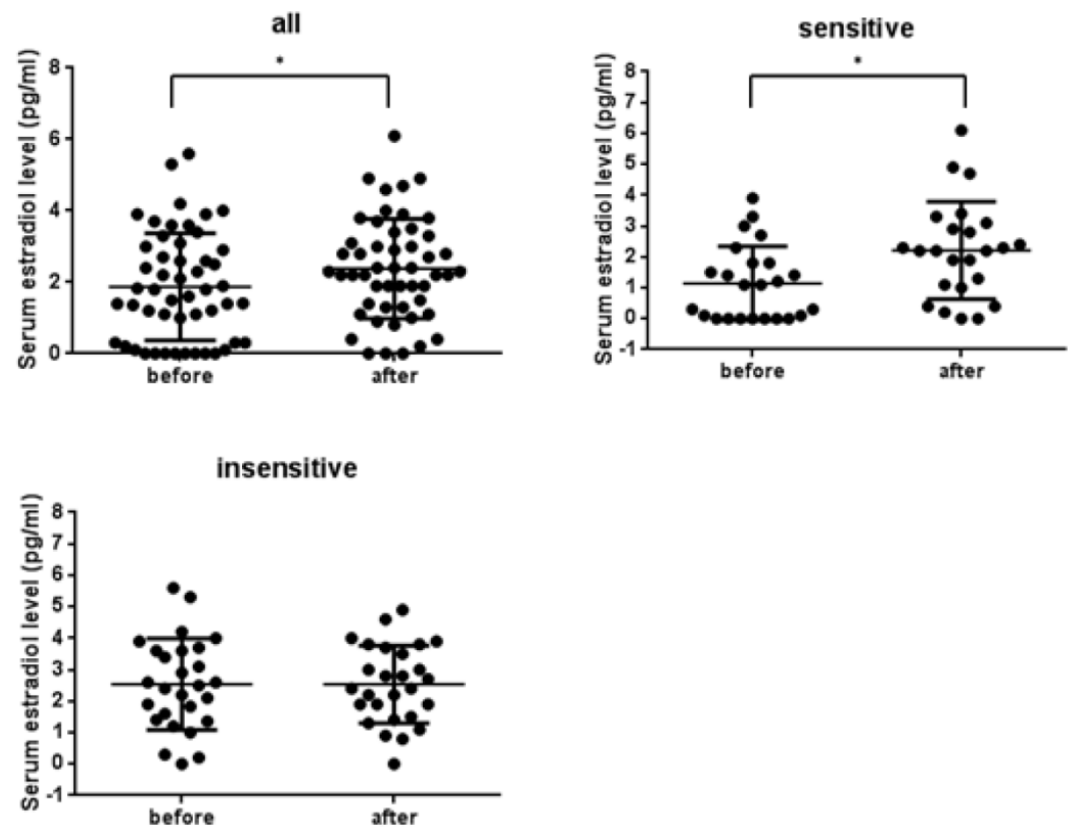

Figure 7. Serum estradiol concentrations in vitamin E-sensitive and insensitive patients who answered all of the questionnaire. 51 patients who answered all questionnaires were divided into vitamin $\mathrm{E}$ sensitive or insensitive groups and their serum estradiol concentrations between before and after vitamin $\mathrm{E}$ administration were compared. In all patients group $(p=0.0412)$ and the sensitive group $(p=0.0124)$, its level was significantly increased after vitamin $E$ administration.
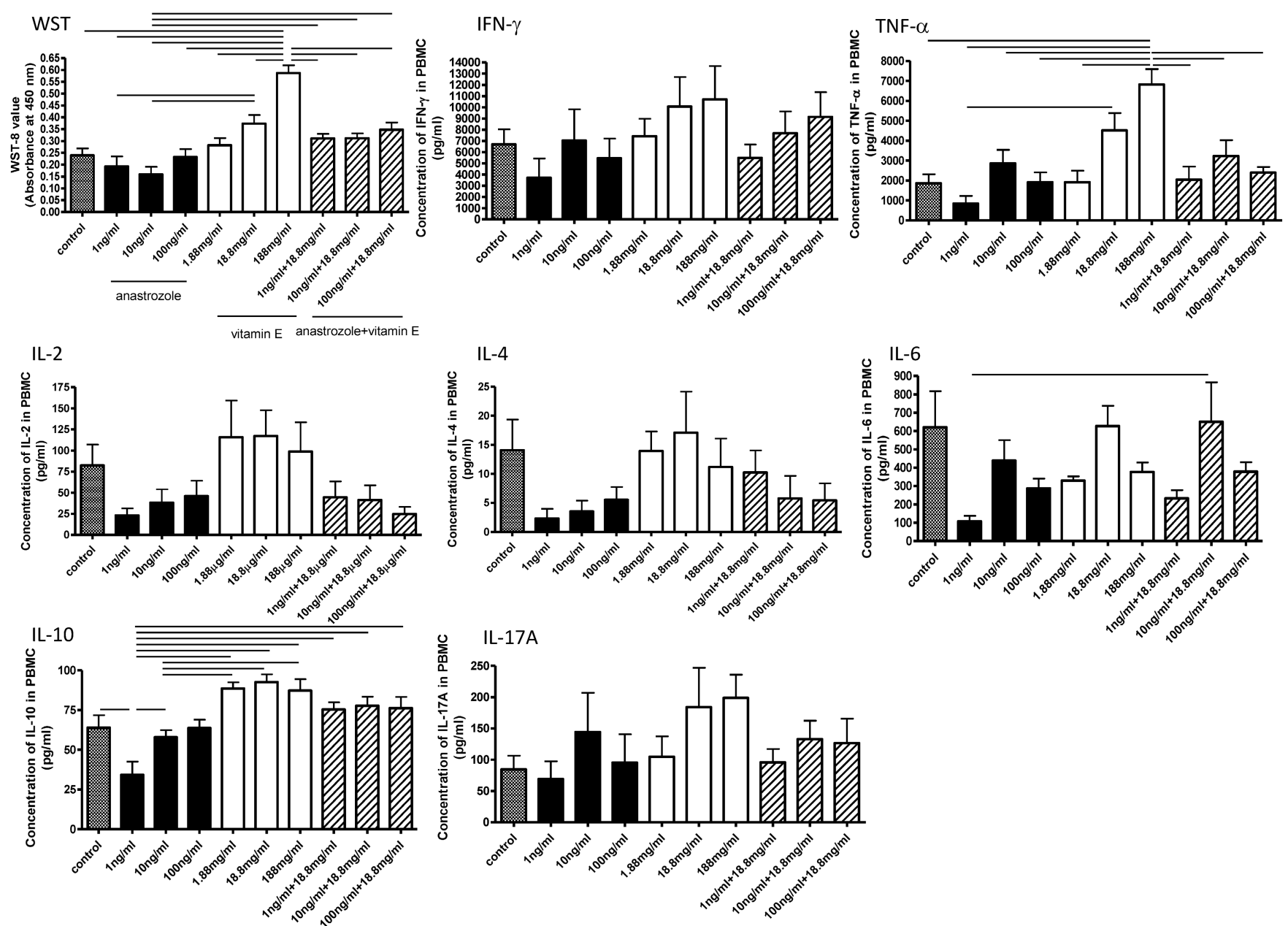

Figure 8. Effects of anastrozole, vitamin E, and their combination on the mitogen-activated PBMCs. PBMCs obtained from health subjects were cultured in the presence of serial concentrations of anastrozole and/or vitamin $E$, under the stimulation with concanavalin $A(n=4)$. Then, WST-8 assay values and the concentrations of IFN- $\gamma$, TNF- $\alpha$, IL-2, IL-4, IL-6, IL-10, and IL-17A in the culture supernatant were measured, and analyzed by Bonferroni's multiple comparison test. The horizontal bars in each figure mean statistical significance between the data $(p<0.05)$. 
In this study, we revealed that there was no significant difference in concentrations of vitamin $\mathrm{E}$, estradiol, progesterone, IFN- $\gamma$, TNF- $\alpha$, IL-2, IL-4, IL-6, and IL-10 between the severe and the mild osteoarthropathy patients (data not shown). These observations suggest that, in the breast cancer patients taking aromatase inhibitors, there is no correlation between the osteoarthropathy severity and the serum biomarkers; vitamin E, estradiol, progesterone, IFN- $\gamma$, TNF- $\alpha$, IL-2, IL-4, IL-6, IL-10, and IL-17A. On the other hand, there was a significant positive correlation between serum estradiol and serum IL-17A concentrations of breast cancer patients taking aromatase inhibitors (Figure 1). Estrogen deficiency was reported to induce differentiation of IL-17 secreting Th17 cells [7]. In addition, another report suggested that IL-17 mediates bone loss in estrogen deficient osteoporotic condition [10]. Anti-inflammatory properties of estrogens include suppression of the effects of IL-17 [11]. Our data (Figure 1) showed positive correlation between these indices, suggesting that estrogen level increases to suppress the inflammatory influence of IL-17. However, in our study, whilst others using mice, we measured the hormones and the cytokine levels in patient's peripheral blood. Therefore, the tissue or organs which produce IL-17 was not clarified, and thus further examinations about mechanism for the correlation between serum estradiol and IL-17A concentrations in the breast cancer patients are required.

Vitamin $\mathrm{E}$ has many functions including antioxidant and anti-inflammatory activities [12]. It is used for the purpose of suppressing peripheral circulatory disturbance in all over the world. On the other hand, there is little report describing the usage of vitamin $\mathrm{E}$ for treatment of osteoarthropathy complicated with aromatase inhibitor. In the present study, we found that administration of vitamin $\mathrm{E}$ for breast cancer patients accompanying osteoarthropathy complicated with aromatase inhibitor use would be an effective treatment in considerable number of patients (Figure 2). We also suggested previously in our pilot study that the administration of vitamin $\mathrm{E}$ is useful in clinical experience as a treatment of osteoarthropathy complicated with aromatase inhibitor in breast cancer patients [3]. Our present data showed that vitamin E administration increased significantly serum vitamin E concentrations (Figure 3), while it did not increase significantly serum estradiol, progesterone, IFN- $\gamma$, TNF- $\alpha$, IL-2, IL-4, IL-6, IL-10, and IL-17A concentrations in the breast cancer patients (data not shown). Thus, the data also suggest that vitamin $\mathrm{E}$ has little unfavorable effect on the efficacy of aromatase inhibitors to suppress breast cancer progression. In human subjects and animal models, high doses of vitamin E were found to exhibit anti-inflammatory effects by decreasing C-reactive protein (CRP) and inhibiting the release of proinflammatory cytokines [13]. Such vitamin E efficacies are also evident in a study on patients with coronary artery disease, whereby the CRP and TNF- $\alpha$ concentrations are found to be significantly lowered with $\alpha$-tocopherol supplementation compared to placebo [14]. In addition, vitamin $E$ is found to inhibit cyclooxygenase-2 activity, which in turn is able to exert anti-inflammatory and anticarcinogenic activities, especially in the colon [15]. On the other hand, it has been suggested that vitamin E stimulates bone resorption and decreases bone mass by inducing osteoclast function [16], and accordingly serum vitamin $\mathrm{E}$ is seemed to be a determinant of bone mass [16]. Controversially, in osteopenic rats induced by ovariectomy, supplementations with either $\alpha$-tocopherol or tocotrienol prevent bone loss and maintained the bone microarchitecture [17]. Further study is required to explore the underlying relationships between estradiol, vitamin $\mathrm{E}$, and function of osteoclast or osteoblast.

There was no significant correlation between serum biomarker concentrations and the osteoarthropathy scores, neither before nor after administration of vitamin E (data not shown). The result suggests that vitamin $\mathrm{E}$ administration decrease osteoarthropathy scores without affecting the serum concentrations of the biomarkers examined in this study. Thus, the severity of osteoarthropathy appears to be independent of serum concentrations of estradiol, progesterone, IFN- $\gamma$, TNF- $\alpha$, IL-2, IL-4, IL-6, IL-10, and IL-17A. Additionally, we suggested in this study that there was no significant influence of aromatase inhibitor and vitamin $\mathrm{E}$ on cytokine production from PBMCs. On the other hand, vitamin E may increase growth potential of $\mathrm{T}$ lymphocytes, when administered in combination with therapeutic dose of anastrozole (Figure 8).

In order to further investigate the effect of vitamin $\mathrm{E}$ administration, we divided 51 patients who answered all questionnaires into sensitive or insensitive group and compared serum estradiol levels. After the administration of vitamin $\mathrm{E}$, the serum estradiol levels were significantly increased in the 51 patients and the vitamin E sensitive group $(\mathrm{n}=24)$, but were not in insensitive patients $(n=27)$ (Figure 7). These observations suggest that serum estradiol level in the vitamin E-sensitive patients may increase by vitamin E administration. However, when we examined changes of serum estradiol concentrations after the vitamin $\mathrm{E}$ administration in the patients, we detected no significant increase in the level. In the selected 51 patients, serum estradiol level changed from 1.88 \pm 
0.21 to $2.38 \pm 0.20 \mathrm{pg} / \mathrm{ml}$, and insensitive patients, it changed from $1.14 \pm 0.25$ to $2.21 \pm 0.32 \mathrm{pg} / \mathrm{ml}$ in the sensitive patients. It has been reported that healthy postmenopausal women have $31.1 \pm 16.64 \mathrm{pg} / \mathrm{ml}$ serum estradiol level [18]. Our patients had been treated by aromatase inhibitor so that their estradiol levels were extremely low, even if after vitamin E administration. From these points of view, there may be no influence of vitamin $\mathrm{E}$ on breast cancer pathology. On the other hand, there is a report describing that vitamin E decreases bone mass by stimulating osteoclast fusion [16]. However, the authors in this report used mice and rats and they didn't examine the implication of sex hormones.

In order to reveal the therapeutic effects of vitamin E for the aromatase inhibitor-induced osteoarthropathy, we also investigated correlations between serum concentrations of several biomarkers and the improvement rates for osteoarthropathy after the vitamin $\mathrm{E}$ administration. We demonstrated from these examinations, that the lower concentration of serum estradiol or IL-17A in breast cancer patients under aromatase inhibitors therapy is related to clinical effectiveness of the vitamin E treatment (Figure 4). It has been reported that IL-17 levels are high in patients with osteoporosis and rheumatism [19]. In osteoclastogenesis of mouse bone marrow-derived macrophages stimulated with the macrophage colony-stimulating factor, IL-17 affects differently on the osteoarthritis depending on the stage [20]. In an early stage, IL-17 appeared to act as an anti-osteoclastogenic factor, while in a late stage, IL-17 enhanced osteoclastogenesis [20]. Especially, challenge with IL-17 in the late phase of experimental osteoclastogenesis in mouse bone marrow-derived macrophages decreased the number of osteoclasts at all given concentrations from 0.1 to $10 \mathrm{ng} / \mathrm{ml}$ in vitro, although the pattern was not dose-dependent [20]. Estrogen deprivation has been reported to promote RANKL expression and bone resorption in association with upregulation of the IL-17 effector, Act1, supporting the concept that post-menopausal osteoporosis is a disorder of innate immunity [21]. The reference value of serum estradiol in postmenopausal women is less than $31.1 \pm 16.64 \mathrm{pg} / \mathrm{ml}$ [18]. In the present study, there was no significant correlation between serum IL-17 concentrations and the osteoarthropathy severity scores before administration of vitamin E. However, after administration of vitamin E, serum IL-17 concentrations correlated significantly with the severity scores. These observations suggest that serum IL-17 level may play a role in severity of osteoarthropathy complicated with aromatase inhibitor treatment. On the other hand, we also experienced the patients whose symptoms became worse after the vitamin E administration. Therefore, it should be noted that we can select out the patients who will respond well to the vitamin $\mathrm{E}$ therapy to improve the symptoms of osteoarthropathy based on the serum IL-17A or estradiol concentration. There are few reports describing about not only efficacy of vitamin $\mathrm{E}$ on osteoarthropathy but also screening of patients who will respond well to vitamin E using any serum biomarkers. Our data suggest that serum estradiol play an important role in improvement of osteoarthropathy and the vitamin E efficacy. The data in the present report showed that, although serum estradiol concentration was not different between the mild and severe osteoarthropathy patients, the concentrations before vitamin $\mathrm{E}$ administration were significantly lower in patients who responded well to the vitamin $\mathrm{E}$ therapy. From these observations, we are convinced that the low concentration of estradiol $(<1.8 \mathrm{pg} / \mathrm{ml})$ could be an useful biomarker for predicting the effectiveness of vitamin E administration for osteoarthropathy complicated with aromatase inhibitor treatment in breast cancer patients.

In conclusion, vitamin $\mathrm{E}$ administration seemed to be a potential way for relieving osteoarthropathy complicated with aromatase inhibitor use. Serum estradiol level of breast cancer patients could be a novel and useful biomarker for predicting the efficacy of vitamin $\mathrm{E}$ treatment for these symptoms. From the established serum estradiol threshold level of $1.8 \mathrm{pg} / \mathrm{ml}$, we would be able to select out the breast cancer patients who will respond well to the pharmacotherapy by vitamin E for the treatment of osteoarthropathy induced by aromatase inhibitor. Further research to reveal the underlying mechanism(s) how vitamin E palliates the osteoarthropathy complicated with aromatase inhibitor would lead vitamin E as efficient and safety agent for supporting the aromatase inhibitor therapy in breast cancer patients.

\section{Conclusions}

Vitamin E administration seemed to be a potential way for relieving osteoarthropathy complicated with aromatase inhibitor use. We concluded that, using serum estradiol level, it would be possible to select out the breast cancer patients who will respond well to the vitamin E therapy for osteoarthropathy complicated with aromatase inhibitor use.

\section{Abbreviations}

IFN- $\gamma$ : interferon- $\gamma$; TNF- $\alpha$ : tumor necrosis factor- $\alpha$; IL: interleukin; ELISA: enzyme-linked immunoassay; QOL: quality of life; ECLIA: electrochemiluminescence immunoassay; CBA: Cytometric Bead Array; ROC: the receiver-operating characteristic; AUC: area under the curve; RANK: receptor-activator 
of nuclear factor $\kappa B$; RANKL: receptor-activator of nuclear factor $\kappa \mathrm{B}$ ligand; Act: actin.

\section{Competing Interests}

There is a financial support of this work from Eisai Inc., Japan.

\section{References}

1. Khanduri S, Dodwell DJ. Aromatase inhibitors and musculoskeletal symptoms. The Breast. 2008; 17: 78-81.

2. Harold J. Burstein. Aromatase inhibitor-associated arthralgia syndrome. The Breast. 2007; 16: 223-234.

3. Makita M, et al. The efficacy of Vitamin E for Aromatase Inhibitor-Associated Arthralgia Syndrome. Japanese Journal of Breast Cancer (in Japanese) 2008; 23(5): 413-416

4. Tappel AL. Lipid Peroxidation Damage to Cell Components. Fed Proc. 1973; 32(8): 1870-4.

5. Williams HTG, et al. Alpha Tocopherol in the Treatment of Intermittent Claudication. Can Med Assoc J. 1962; 87(10): 538-541.

6. Pfeilschifter J, Kodits R, Pfohl M, Schatz H. Changes in Proinflammatory Cytokine Activity after Menopause. Endocr Rev. 2002; 23(1): 90-119.

7. Tyagi AM, Srivastava K, Mansoori MN, Trivedi R, Chattopadhyay N, Singh D. Estrogen deficiency induces the differentiation of IL-17 secreting Th17 cells: a new candidate in the pathogenesis of osteoporosis. PLoS ONE. 2012; 7(9): e44552. doi: 10.1371/journal.pone.0044552.

8. Pusztai L, Mendoza TR, Reuben JM, Martinez MM, Willey JS, Lara J, Syed A, Fritsche HA, Bruera E, Booser D, Valero V, Arun B, Ibrahim N, Rivera E, Royce M, Cleeland CS, Hortobagyi GN. Changes in plasma levels of inflammatory cytokines in response to paclitaxel chemotherapy. Cytokine 2004; 25: 94-102

9. Nakamura T, Imai $Y$, Matsumoto T, Sato S, Takeuchi K, Igarashi K, Harada Y, Azuma Y, Krust A, Yamamoto Y, Nishina H, Takeda S, Takayanagi H, Metzger D, Kanno J, Takaoka K, Martin TJ, Chambon P, Kato S. Estrogen Prevents Bone Loss via Estrogen Receptor $\alpha$ and Induction of Fas Ligand in Osteoclasts. Cell. 2007; 130(5): 811-823.

10. DeSelm CJ, Takahata Y, Warren J, Chappel JC, Khan T, Li X, Liu C, Choi Y, Kim YF, Zou W, Teitelbaum SL. IL-17 mediates estrogen-deficient osteoporosis in an Act1-dependent manner. J Cell Biochem. 2012; 113(9): 2895-2902.

11. Straub RH. The complex role of estrogens in inflammation. Endocr Rev. 2007; 28(5): 521-574

12. Salinthone S, Kerns AR, Tsang V, Carr DW. $\alpha$-Tocopherol (vitamin E) stimulates cyclic AMP production in human peripheral mononuclear cells and alters immune function. Mol Immunol. 2013; 53(3): 173-178.

13. Singh U, Devarai S. Vitamin E: inflammation and atherosclerosis. Vitam Horm. 2007; 76: 519-549

14. Devarai S, Tang R, Adams-Huet B, Harris A, Seenivasan T, de Lemos JA, Jialal I. Effect of high-dose $\alpha$-tocopherol supplementation on biomarkers of oxidative stress and inflammation and carotid atherosclerosis in patients with coronary artery disease. Am J Clin Nutr, 2007; 86(5): 1392-8

15. Qing Jiang, Xinmin Yin, Markus A. Lill, Matthew L. Danielson, Helene Freisher, Jianjie Huang. Long-chain carboxychromanols, metabolites of vitamin E, are potent inhibitors of cyclooxygenases. Proc Natl Acad Sci USA, 2008; 105(51): 20464-9

16. Fujita K, Iwasaki M, Ochi H, Fukuda T, Ma C, Miyamoto T, Taketani K, Negishi-Koga T, Sunamura S, Kodama T, Takayanagi H, Tamai H, Kato S, Arai H, Shinomiya K, Itoh H, Okawa A, Takeda S. Vitamin E decreases bone mass by stimulating osteoclast function. Nat Med, 2012; 18(4): 589-94. doi: $10.1038 / \mathrm{nm} .2659$.

17. Muhammad N, Luke DA, Shid AN, Mohamed N, Soelaiman IN. Two different isomers of vitamin E prevent bone loss in postmenopausal osteoporosis rat model. Evid Based Complement Altemat Med, 2012: 161527. doi: $10.1155 / 2012 / 161527$.

18. Zhao Z, Mai Z, Ou L, Duan X, Zeng G. Serum Estradiol and Testosterone Levels in Kidney Stones Disease with and without Calcium Oxalate Components in Naturally Postmenopausal Women. PLoS One. 2013; 8(9): e75513.

19. Pickens SR, Volin MV, Mandelin AM II, Kolls JK, Pope RM, Shahrara S. IL-17 contributes to angiogenesis in rheumatoid arthritis. J Immunol. 2010; 184(6): 3233-41

20. Moon SJ, Ahn IE, Jung H, Yi H, Kim J, Kim Y, Kwok SK, Park KS, Min JK, Park $\mathrm{SH}, \mathrm{Kim} \mathrm{HY}$, Ju JH. Temporal differential effects of proinflammatory cytokines on osteoclastogenesis. Int J Mol Med. 2013; 31(4): 769-777.

21. DeSelm CJ, Takahata Y, Warren J, Chappel JC, Khan T, Li X, Liu C, Choi Y, Kim YF, Zou W, Teitelbaum SL. IL-17 mediates estrogen-deficient osteoporosis in an Act1-dependent manner. J Cell Biochem. 2012; 113(9): 2895-2902. 